\title{
Thymidylate synthase (TS) immunostaining in the diagnosis of the myoepithelial cells, basal cells, stratified epithelium cells, and associated tumors
}

\author{
Rui Guo ${ }^{1}$, Yi Tian ${ }^{1}$, Na Zhang ${ }^{1}$, Hong Huang ${ }^{1}$, Ying Huang ${ }^{1}$, Xueyuan Jin ${ }^{1}$, Xiaozhong Huang ${ }^{1}$, \\ Zongfang $\mathrm{Li}^{1,2}$, Jun Yang \\ ${ }^{1}$ Department of Pathology, The Second Affiliated Hospital, Xi'an Jiaotong University, The Second Affiliated Hospital, Xi'an 710004, China; \\ ${ }^{2}$ National-Local Joint Engineering Research Center of Biodiagnostics and Biotherapy, Xi'an 710004, China \\ Contributions: (I) Conception and design: R Guo, Z Li, J Yang; (II) Administrative support: None; (III) Provision of study materials or patients: \\ Y Tian, Z Na, H Huang; (IV) Collection and assembly of data: Y Huang, X Jin, X Huang; (V) Data analysis and interpretation: R Guo, Q Yu, N \\ Huang, H Huang, J Yang; (VI) Manuscript writing: All authors; (VII) Final approval of manuscript: All authors. \\ Correspondence to: Jun Yang, PhD, MD. Department of Pathology, Xi'an Jiaotong University, Second Affiliated Hospital, No. 157, The West $5^{\text {th }}$ Road, \\ Xi'an 710004, China. Email: yangjundr@163.com.
}

Background: Thymidylate synthase (TS) is an important prognostic biomarker for resistance to 5FUbased adjuvant chemotherapy. Recently, we found that TS was specifically expressed in the nucleus of the myoepithelial cell (MEC), basal cell (BC), transitional epithelial cell (TEC), squamous epithelial cell (SEC), and associated tumor using immunostaining. This prompted us to examine whether TS could be used as a diagnostic biomarker for MEC, BC, TEC, SEC, and associated tumors.

Methods: Formalin-fixed, paraffin-embedded specimens from 186 cases of tumors were immunostaining for expression of TS and p63. The diagnostic capability of TS as a reliable diagnostic marker was evaluated and compared with the expression of p63.

Results: TS exhibited a strong specific and stable nuclear immunoreactivity in all specimens including MEC, BC, TEC, and SEC when compared with p63. Notably, a variable degree of TS cytoplasmic positive immunoreactivity was observed in $58.3 \%$ of squamous-cell carcinoma (SQCC), $37.5 \%$ of basal cell carcinoma (BCC), 44.4\% transitional-cell carcinomas (TCC), 41.7\% mixed tumor (MT) and 56.5\% of adenocarcinoma (ADC) specimens.

Conclusions: In addition to being used as a strong prognostic factor for 5-FU resistance, TS also serves as a promising putative diagnostic marker for identifying MEC, BC, SEC, and TEC from GEC, and for distinguishing SQCC, BCC, TCC, and MT from ADC.

Keywords: Basal cell (BC); immunohistochemistry (IHC); myoepithelial cell (MEC); stratified epithelium; thymidylate synthase (TS)

Submitted Jul 30, 2019. Accepted for publication Nov 15, 2019.

doi: $10.21037 /$ tcr.2019.12.18

View this article at: http://dx.doi.org/10.21037/tcr.2019.12.18

\section{Introduction}

The unprecedented advances in molecular testing and personalized therapy have posed emerging challenges for pathologist attributed to the rapidly changing landscape of oncologic pathology. Moreover, these conceptual changes have exponentially improved our understanding of molecular complexities based on the phenotypic and molecular characteristics of cancer. Thus, identification of molecular subsets of carcinomas is crucial to establish a diagnosis, and contribute to prognostication, therapeutic decisions, and determine appropriate targeted molecular strategies for personalized patient care $(1,2)$. 
There is a group tumors that originates from squamous epithelial cell (SEC), basal cell (BC), transitional epithelial cell (TEC) and myoepithelial cell (MEC) in various organs, including the squamous-cell carcinoma (SQCC), basal cell carcinoma (BCC), transitional-cell carcinoma (TCC), mixed tumor (MT), and other types of epithelial myoepithelial tumor (EMT) (3-6). These are the most frequently diagnosed neoplasms and the most frequent cause of major cancer incidence worldwide. Thus, it is becoming increasingly crucial to distinguish the abovementioned tumors from adenocarcinoma (ADC), particularly in the present era of targeted molecular therapies $(7,8)$. Notably, accurate subtyping is essential for the stratification of therapeutic drugs, as only certain chemotherapeutic drugs are acceptable while others are contraindicated in tumor lesions.

Generally, in most cases, histologic subtyping can be reliably performed with hematoxylin-eosin (H\&E)stained slides alone. However, in a substantial proportion of problem cases, particularly in small biopsy specimens or poorly differentiated tumors, standard morphology alone is insufficient to appropriately classify the subtypes and warrants further histopathological characterization. Thus, distinguishing cancer subtypes can often present a formidable challenge to the pathologist (9-12).

To overcome these challenges, immunohistochemical staining is usually employed to identify markers associated with tumor subtypes to assist the final histologic diagnosis of the tumor. In the past, numerous immunohistochemical markers have been reported to be useful in making the final diagnosis of the tumor, and many markers gained acceptance and translated into routine usage (13-16). Among them, a combination of cytokeratin 5 and 6 (CK5/6) and $\mathrm{p} 63$, and $\mathrm{p} 40$ immunohistochemical staining coupled with histological findings could be easily applied and could reliably distinguish SQCC, BCC, TCC and MT from ADC and other subtypes of the tumor $(17,18)$.

Thymidylate synthase (TS) is an important prognostic biomarker for resistance to $5 \mathrm{FU}$-based adjuvant chemotherapy (19). Accumulating evidence has indicated that 5-Fluorouracil (5-FU) predominantly exerts its antitumor effects through inhibition of TS. However, over-expression of TS has been noticeably associated with the resistance to 5FU-based adjuvant chemotherapy $(20,21)$. Consequently, TS expression has been utilized as a biomarker for $5-\mathrm{FU}$ resistance in various malignant tumor with 5-FU-based chemotherapy (22). Recently, while investigating the clinical correlation between the expression of TS and 5-FU resistance, we observed that TS was explicitly expressed in the nucleus of MEC, BC, SQC, and TEC and other associated neoplasms derived from the above-mentioned cells using immunostaining (23).

Based on these novel findings, we hypothesized that TS may possibly be used as a putative biomarker for identification of SEC, BC, TEC, and MEC, and for the differential diagnosis of SQCC, BCC, TCC, and MT from $\mathrm{ADC}$ and other tumor subtypes. Furthermore, in the present study we evaluated the diagnostic utility of TS as a promising biomarker for the identification of SEC, BC, TEC, and MEC and the differential diagnosis of SQCC, BCC, TCC, and MT from ADC in 186 cases of neoplasms.

\section{Methods}

\section{Clinical specimens and materials}

A total of 186 cases of well- and moderately-differentiated tumor specimens were retrieved from archives of the Department of Pathology, of the Second Hospital, Xi'an Jiaotong University (Xi'an, China) from 2017 to 2019. The specimens included were 63 cases of SQCC, 8 cases of BBC, 18 cases of TCC, 12 cases of MT and 85 cases of ADC. Tumor tissues were collected from biopsies and surgical resections of the breast, nasal cavity and paranasal sinus, throat and pharynx, lung, salivary gland, esophagus, stomach and colon, ureters and bladder, prostate, cervix, and skin (Table 1). The specimens were fixed in $10 \%$ neutral buffered formalin and embedded in paraffin. Hematoxylin and eosin (H\&E)-stained tissue sections of all specimens were reviewed by two qualified pathologists according to the 2015 World Health Organization (WHO) histological Classification system. All cases were annotated with accessible clinical data to ensure the complete privacy and confidentiality of patient identity. This study was approved by the Second hospital Review Board of Xi'an Jiaotong University (Xi'an, China).

\section{Reagents and instruments}

Anti-TS antibody (clone TS106, dilution 1:100) was purchased from Guangzhou AmBiPing (LBP) Pharmaceutical Technology Co., Ltd (Guangzhou, China). Anti-p63 (clone MX013, dilution 1:100) was purchased from Maixin Biotechnology Development Co., Ltd (Fuzhou, China). UltraView Universal DAB Detection Kit and Ventana Benchmark XT Automated Slide Stainer (Ventana 
Table 1 TS and p63 expression in normal cells from various organs and tissues

\begin{tabular}{|c|c|c|c|c|c|}
\hline \multirow{2}{*}{ Organ } & \multirow{2}{*}{ Cell type } & \multirow{2}{*}{$\begin{array}{l}\text { Number of } \\
\text { cases }(\mathrm{N})\end{array}$} & \multicolumn{3}{|c|}{ Immunopositive, n/N (\%) } \\
\hline & & & Nuclear staining & Cytoplasmic staining* & $\frac{\mathrm{p} 63}{\text { Nuclear staining }}$ \\
\hline \multirow[t]{2}{*}{ Breast } & GEC & 26 & $0 / 26[0]$ & $2 / 26(7.7)$ & $0 / 26[0]$ \\
\hline & MEC & 26 & $26 / 26[100]$ & $0 / 26[0]$ & $26 / 26[100]$ \\
\hline \multirow{2}{*}{$\begin{array}{l}\text { Nasal cavity \& paranasal } \\
\text { sinus }\end{array}$} & GEC & 7 & 0/7 [0] & 0/7 [0] & 0/7 [0] \\
\hline & $\mathrm{BC}$ & 7 & $7 / 7$ [100] & 0/7 [0] & $7 / 7$ [100] \\
\hline \multirow[t]{2}{*}{ Throat \& pharynx } & SEC & 6 & 6/6 [100] & 0/6 [0] & 6/6 [100] \\
\hline & GEC & 6 & $0 / 6[0]$ & $0 / 6[0]$ & $0 / 6[0]$ \\
\hline \multirow{2}{*}{ Lung } & GEC & 39 & 0/39 [0] & 2/39 (5.1) & $0 / 39[0]$ \\
\hline & AEC & 39 & $0 / 39[0]$ & $3 / 39(7.7)$ & $0 / 39[0]$ \\
\hline \multirow[t]{2}{*}{ Salivary gland } & GEC & 12 & 0/12 [0] & $1 / 12(8.3)$ & 0/12 [0] \\
\hline & MEC & 12 & $12 / 12[100]$ & $0 / 12[0]$ & $12 / 12[100]$ \\
\hline esophagus & SEC & 9 & 9/9 [100] & $0 / 9[0]$ & $9 / 9[100]$ \\
\hline Stomach \& colon & GEC & 23 & $0 / 23[0]$ & $2 / 23(8.3)$ & $0 / 23[0]$ \\
\hline Ureters \& bladder & TEC & 18 & 18/18 [100] & 0/18 [0] & 18/18 [100] \\
\hline Prostate & GEC & 15 & 0/15 [0] & $0 / 15(6.7)$ & 0/15 [0] \\
\hline Skin & MEC & 17 & $17 / 17$ [100] & 0/17 [0] & $17 / 17$ [100] \\
\hline
\end{tabular}

GEC, glandular epithelial cell; MEC, myoepithelial cells; SEC, squamous epithelial cell; TEC, transitional epithelial cell; BC, basal cell. AEC, alveolar epithelia. * , weak cytoplasmic positive immunostaining for TS was observed in few GECs or AEC.

Medical Systems, Roche, Shanghai, China) were used in staining procedure.

\section{Immunobistochemical analysis}

For immunohistochemical analysis, paraffin-embedded tissues were serially sectioned $(4-5 \mu \mathrm{m})$, heated at $58{ }^{\circ} \mathrm{C}$ for 1 hour, and subjected to immunohistochemistry (IHC). Immunostaining was performed using a standard immunostaining protocol using a Venta Benchmark XT
Automated Stainer. Briefly, the sections were deparaffinized in xylene, rehydrated in graded ethanol series, and endogenous peroxidase activity was inhibited with $5 \%$ hydrogen peroxide. Then, sections were blocked with normal goat serum. Antigen retrieval was performed using the standard procedure with a Ventana Benchmark XT Automated EDTA method for 30 minutes in $1 \mathrm{mM}$ EDTA (pH 8.0). Then, sections were incubated with antiTS and anti-p63 primary antibodies, respectively, for 30 min at $37^{\circ} \mathrm{C}$. Subsequently, after washing three times 
Table 2 TS and p63 expression in various tumor cells

\begin{tabular}{|c|c|c|c|c|c|}
\hline \multirow{2}{*}{ Organ } & \multirow{2}{*}{ Tumor type } & \multirow{2}{*}{$\begin{array}{c}\text { Number of } \\
\text { cases }(\mathrm{N})\end{array}$} & \multicolumn{3}{|c|}{ Immunopositive, n/N (\%) } \\
\hline & & & Nuclear staining & Cytoplasmic staining ${ }^{\#}$ & $\begin{array}{c}\text { p63 } \\
\text { Nuclear staining }\end{array}$ \\
\hline Breast & ADC & 26 & $0 / 26[0]$ & $14 / 26(53.8)$ & $0 / 26[0]$ \\
\hline Nasal cavity \& paranasal sinus & SQCC & 7 & $7 / 7[100]$ & $3 / 7(42.6)$ & $7 / 7$ [100] \\
\hline \multirow[t]{2}{*}{ Lung } & SQCC & 18 & $18 / 18[100]$ & $11 / 18(61.1)$ & $18 / 18[100]$ \\
\hline & ADC & 21 & $0 / 21[0]$ & 13/21 (61.9) & $0 / 21[0]$ \\
\hline Salivary gland & MT & 12 & $12 / 12[100]$ & $5 / 12(41.7)$ & 12/12 [100] \\
\hline esophagus & SQCC & 9 & 9/9 [100] & $3 / 9(33.3)$ & 9/9 [100] \\
\hline Prostate & ADC & 15 & $0 / 15[0]$ & $9 / 15(60.0)$ & $0 / 15[0]$ \\
\hline Cervix & SQCC & 14 & $14 / 14[100]$ & 6/14 (42.9) & $14 / 14[100]$ \\
\hline \multirow[t]{2}{*}{ Skin } & SQCC & 9 & 9/9 [100] & $3 / 9(33.3)$ & 9/9 [100] \\
\hline & $\mathrm{BCC}$ & 8 & $8 / 8[100]$ & $3 / 8(37.5)$ & 8/8 [100] \\
\hline
\end{tabular}

in Tris-buffered saline (TBS), sections were incubated with horseradish peroxidase-conjugated secondary antibody for 15 minutes at $37^{\circ} \mathrm{C}$. Sections were then treated with 3,3'-diaminobenzidine chromogen (DAB; Roche, Shanghai, China) for 8 minutes, and then the sections were counterstained with Mayer's hematoxylin, dehydrated in alcohol, cleared with xylenes, and mounted. Simultaneously, the sections of SEC from nonneoplastic skin tissue were employed as a positive control. As a negative control, the primary antibody was replaced with phosphate-buffered saline.

\section{IHC interpretation}

TS or p63 expression in tumor cells and SEC or BC or TEC or MEC or AEC in para-carcinoma tissue was evaluated in all 186 tumor specimens. The HE-stained and immunostaining sections were reviewed independently by two pathologists to obtain a consensual score. The extent of the nuclear staining was expressed as a percentage of the positive cell for TS or p63 staining from 0 to $100 \%$, with increments of $10 \%$. Using $10 \%$ as the cut-off value, sections with a more than $10 \%$ expression were considered to exhibit positive TS or p63 expression. The intensity of cytoplasmic TS immunostaining in cells was assessed and scored on a four-point scale from negative $(0)$, weak $(+1)$, moderate $(+2)$, and strong intensity $(+3)$.

\section{Statistical analysis}

Using pathological classification as the gold standard, the sensitivity and specificity were calculated for TS and p63 markers. Data were expressed as total numbers and percentages. Chi-square-tests and Fisher's exact tests were performed to compare the frequencies of positive and negative diagnoses. Pearson's correlation was used to determine the association between the expression of TS and $\mathrm{p} 63$. Significance levels were set at $5 \%$ for all tests. Statistical analyses were performed using the SPSS 17 software (SPSS Inc., Chicago, IL, USA). P values $<0.05$ were considered statistically significant.

\section{Results}

The results of TS and p63 expression in 186 tumor specimens are summarized in Tables 1-3. 
Table 3 TS and p63 expression in various tumor types

\begin{tabular}{|c|c|c|c|c|}
\hline \multirow{2}{*}{ Tumor type } & \multirow{2}{*}{$\begin{array}{l}\text { Number of cases } \\
(\mathrm{N})\end{array}$} & \multicolumn{3}{|c|}{ Immunopositive, $\mathrm{n} / \mathrm{N}(\%)$} \\
\hline & & Nuclear staining & Cytoplasmic staining ${ }^{\#}$ & $\frac{\text { p63 }}{\text { Nuclear staining }}$ \\
\hline SQCC & 63 & 63/63 [100] & 25/63 (39.7) & $63 / 63[100]$ \\
\hline $\mathrm{BCC}$ & 8 & $8 / 8[100]$ & $3 / 8(37.5)$ & $8 / 8[100]$ \\
\hline MT & 12 & $12 / 12[100]$ & $5 / 12(41.7)$ & $12 / 12[100]$ \\
\hline ADC & 85 & $0 / 85[100]$ & 48/85 (56.5) & 0/85 [100] \\
\hline
\end{tabular}

", cytoplasmic variable positive of TS staining was showed in tumor cells of some tumor specimens. SQCC, squamous-cell carcinoma; ADC, adenocarcinoma; BCC, basal cell carcinoma; TCC, transitional cell carcinoma; MT, mixed tumor.

\section{TS and p63 expression in nonneoplastic skin tissue}

In nonneoplastic skin tissue sections, both the TS and p63 were specifically and stably expressed in nucleus of SEC, BC, TEC, MEC of breast, nasal cavity and paranasal sinus, throat and pharynx, lung, salivary gland, esophagus, ureters and bladder, prostate, cervix, and skin specimens, respectively (Table 1 and Figure 1).

In contrast, a negative expression of TS and p63 was observed in nuclear of all glandular epithelial cells (GEC) or alveolar epithelial cell (AEC) of the breast, nasal cavity and paranasal sinus, throat, and pharynx, salivary gland, stomach and colon, prostate, cervix, and skin specimens.

Intermittently, TS exhibited a weak positive expression in the cytoplasmic of few GEC or AEC (0-8.3\%) of breast, nasal cavity and paranasal sinus, throat and pharynx, salivary gland, stomach and colon, prostate, cervix, and skin specimens with absence of p63 immunostaining in the cytoplasm (Table 1).

\section{TS and p63 expression in neoplastic tissue}

TS and p63 immunostaining exhibited a consistent nuclear pattern with strong immunoreactivity in nucleus of tumor cells of SQCC (63/63), BCC (8/8), TCC (18/18), and neoplastic MECs of MT (12/12) from nasal cavity and paranasal sinus, throat and pharynx, lung, salivary gland, esophagus, ureters and bladder, cervix and skin specimens, but not in the nucleus of tumor cells from ADC (0/85) and with negative immunostaining for p63 expression in the cytoplasm (Tables 2,3, and Figure 2).

Furthermore, a variable degree of TS cytoplasmic positive immunoreactivity was observed in $58.3 \%$ of SQCC,
$37.5 \%$ of BCC, $44.4 \%$ of TCC, $41.7 \%$ of MT and $56.5 \%$ of ADC specimens (Tables 2,3).

However, in the present study, no differences in TS and p63 expression in the nucleus of SEC or BC or TEC or MEC or GEC or AEC and the associated tumor were observed (Table 4).

\section{TS and p63 expression in other types of cells}

Furthermore, TS was also variably positively expressed in the cytoplasm of few stromal cells and lymphocytes scattered throughout tumors tissue from the breast, nasal cavity and paranasal sinus, throat and pharynx, salivary gland, stomach and colon, prostate, cervix and skin specimens, with the absence of $\mathrm{p} 63$ expression.

\section{Discussion}

TS is the critical enzyme that catalyzes the methylation of dUMP to dTMP with 5,10-methylenetetrahydrofolate as a cofactor. Therefore, it provides the intracellular de novo source of thymidylate, which is essentially required for DNA replication and repair (24). Studies have shown that overexpression of TS is believed to confer resistance to $5 \mathrm{FU}$-based adjuvant chemotherapy, and has been considerably associated with the poor prognosis of the subset of aggressive solid tumors, including breast, lung, stomach, and colorectal cancer $(25,26)$. Moreover, in many clinical studies, a significant positive correlation was observed between overexpression of TS and resistance to 5 -FU in various malignant tumor using 5 -FU-based chemotherapy $(27,28)$. Thus, TS overexpression is used 

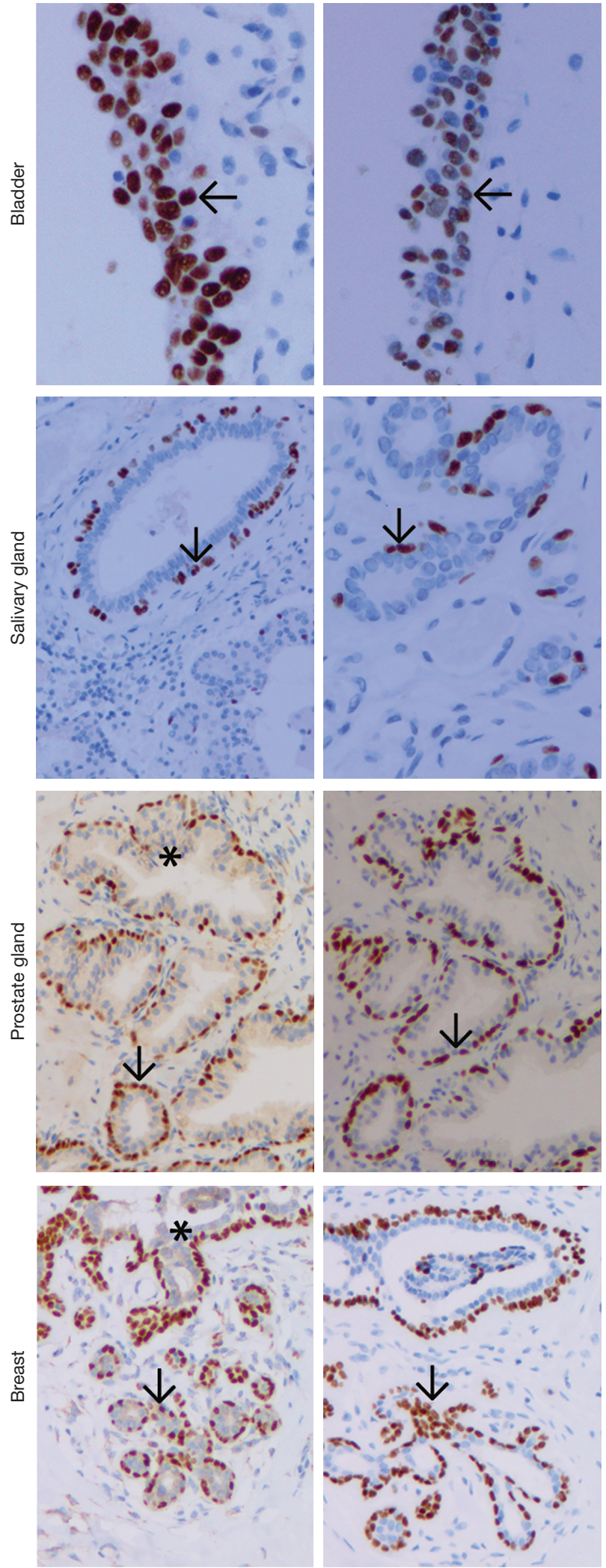

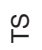
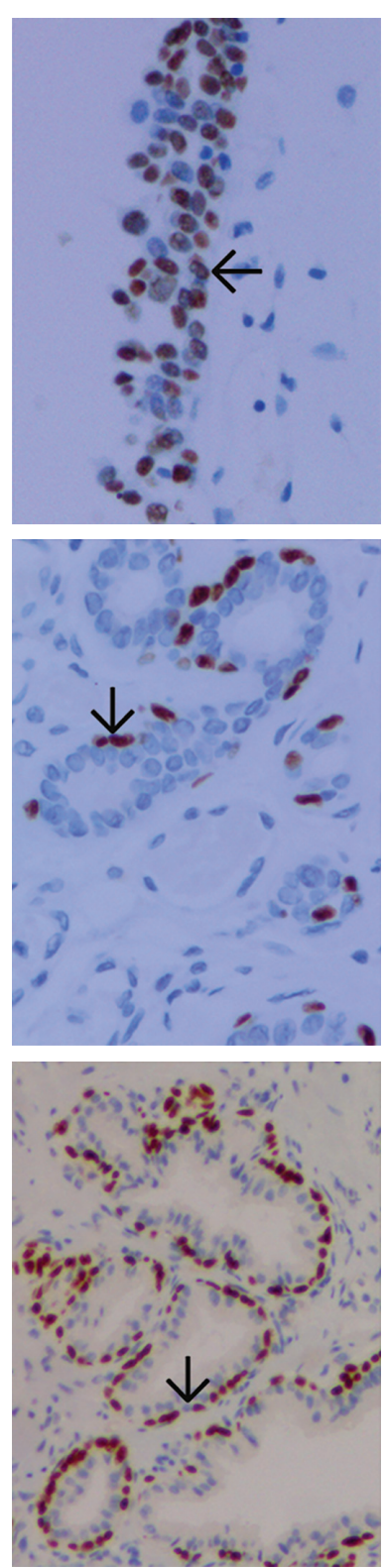

ஜ
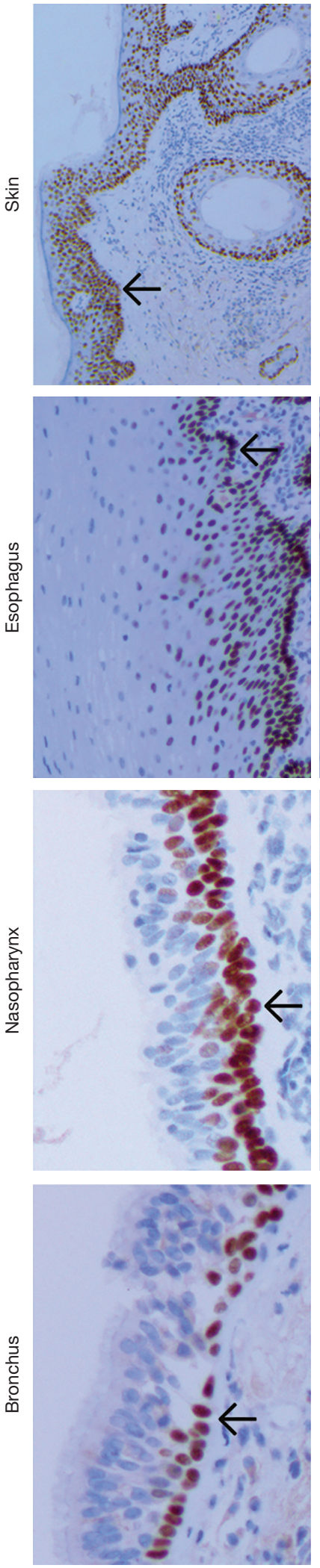

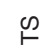

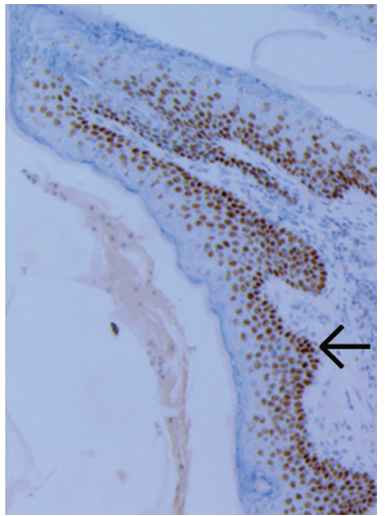

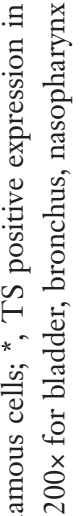
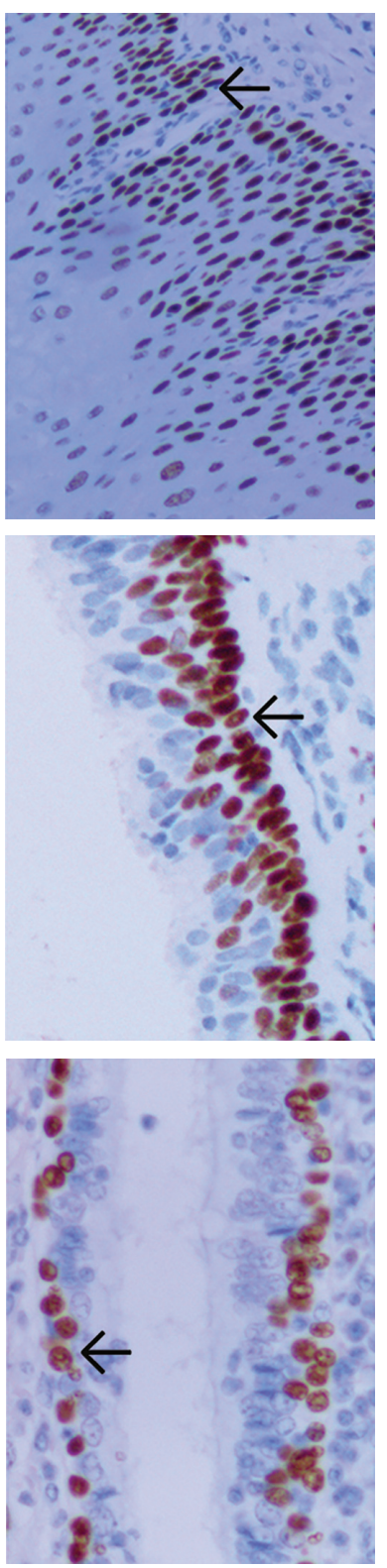

ஜ묘

$\exists$.
芯

5
0
$\overline{0}$
$\overline{0}$

플

.

荶

t) 8

氖

西

茴

$\leftarrow: \frac{\pi}{\pi}$

छ

.$\frac{\pi}{6}$

芞

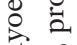

实密

$\downarrow \stackrel{0}{0}$

気

के है

氙

:

을

青安

可

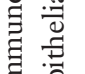

ถุ

声

की

긍

t) 

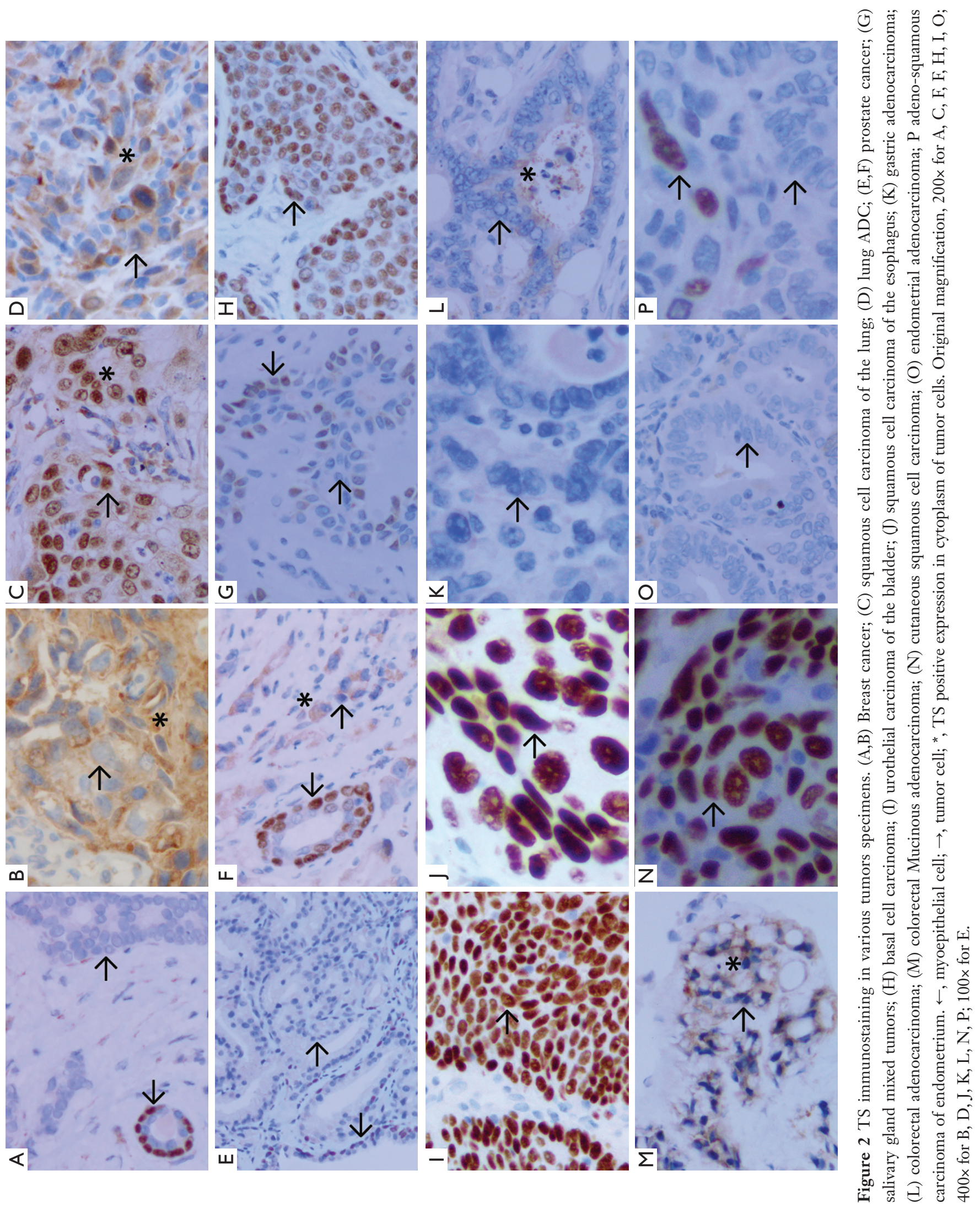
Table 4 Significance of TS and p63 immunostaining in carcinoma

\begin{tabular}{|c|c|c|c|c|c|c|c|c|c|c|}
\hline $\begin{array}{l}\text { Staining } \\
\text { antigens }\end{array}$ & \multicolumn{5}{|c|}{ Cancer type +/total (\%) } & Sen & Spe & PPV & NPV & Accuracy \\
\hline TS/p63+ & 63 & 8 & 18 & 12 & 0 & $100 \%$ & $100 \%$ & $100 \%$ & $100 \%$ & $100 \%$ \\
\hline TS/p63- & 0 & 0 & 0 & 0 & 85 & & & & & \\
\hline
\end{tabular}

SQCC, squamous-cell carcinoma; ADC, adenocarcinoma; BCC, basal cell carcinoma; TCC, transitional cell carcinoma; MT, mixed tumor. SEN, sensitivity; SPE, specificity; PPV, positive predictive value; NPV, negative predictive value; ACC, accuracy.

as an important independent prognostic factor and a biomarker for evaluating the response or resistance to fluoropyrimidine-based chemotherapy $(22,29,30)$.

Previously, we observed that TS was specifically expressed in the nucleus of MEC, BC, TEC, SEC, and tumor cells of SQCC, TCC, BCC, and myoepithelial neoplasm. Therefore, in this study, we proposed to evaluate the diagnostic utility of TS as a highly sensitive and specific marker for detection of SEC, BC, TEC, MEC, and differentiation of SQCC, BCC, TCC, and MT from ADC.

To validate the significance of TS expression as a diagnostic marker, a total of 189 cases of well or moderately differentiated tumor specimens from surgical resection were collected for the study. Histological diagnosis was based on routine $\mathrm{H} \& \mathrm{E}$-stained sections as the gold standard. Furthermore, the sensitivity and specificity of TS expression were compared to those of $\mathrm{p} 63$ by analyzing their expression in all tumor specimens.

The results revealed that the staining patterns, frequency, and overall sensitivity and specificity of TS expression were consistent with that of p63 expression, which is consistent with data reported previously. However, there were no statistically significant differences between the nuclear pattern of TS immunoreactivity and those of p63 for the accurate identification of MEC, BC, SEC, TEC from GEC, and for distinguishing SQCC, BCC, TCC, and MT from ADC.

Simultaneously, our data further indicated that TS and p63 all exhibited high sensitivity, specificity, PPV, and NPV (i.e., high diagnostic accuracy). TS staining exhibited 100\% sensitivity and specificity for SEC, BC, TEC, MEC and SQCC, BCC, TCC, and MT from various organs (Table 4). This may be attributed to the fact that all cases in this group were well- and moderately-differentiated tumors. However, these data suggested that TS expression levels could be used as a novel valuable marker for the detection of the spectrum of tumors, including MEC, BC, SEC, TEC, and SQCC, TCC, BCC, MT, which are both accurate and consistent with p63 expression. Therefore, prospectively, the sensitivity and specificity of TS expression in poorly differentiated tumors must be evaluated as an ideal marker. Furthermore, the nuclear expression pattern of TS demonstrated that TS expression might possibly play an important role in cellular differentiation of stratified epithelium, including, squamous epithelium, and transitional epithelium. However, this fact warrants further investigation in future research.

Furthermore, TS exhibited a weak positive cytoplasmic expression in a few normal epithelial cells from various organs. Particularly, variable positive cytoplasmic expression of TS was observed in tumor cells of SQCC, BCC, TCC, $\mathrm{MT}$, and $\mathrm{ADC}$, with varying degrees of positive staining intensity. Conversely, negative expression of p63 was detected in the cytoplasm of all the cells. Taken together, these studies suggested that TS expression is an important prognostic marker. Besides, the positive TS immunostaining was significantly correlated with favorable prognosis of tumors. The positive cytoplasmic TS expression level in tumor cells was also positively correlated with the response to drug resistance to 5FU-based chemotherapy (31).

There were several limitations to our study. Mainly, to guarantee diagnostic accuracy and function as a gold standard for assessing the specificity and sensitivity of TS immunostaining, the majority of tumors specimens included in this study were from sufficiently large surgical specimens with well or moderately differentiated tumor cells. Therefore, further studies on a large series of small biopsy or poorly differentiated specimens originating from different organs and tissues are warranted to evaluate the specificity and sensitivity of TS as a reliable diagnostic marker. Furthermore, to better characterize the underlying mechanism and function of TS expression in MEC, BC, SEC, TEC development, and associated tumor differentiation further investigations are highly desirable.

In summary, in addition to being used as a strong prognostic marker for drug resistance to 5-FU-based chemotherapeutic drugs in tumor patients with a 
cytoplasmic TS overexpression, TS can serve as a promising putative diagnostic marker for the detection of SEC, BC, TEC, MEC from GEC, and for distinguishing SQCC, BCC, TCC MT from ADC. Thus, TS can function as a dual-functional molecular marker. Moreover, the most notable from these diagnostic and prognostic data is the fact that these can be obtained in a single immunostaining procedure, which is the key advantage and major characteristic of TS expression compared with $\mathrm{p} 63$.

\section{Acknowledgments}

We thank mogoedit (http://www.mogoedit.com) for editing a draft of this manuscript.

Funding: None.

\section{Footnote}

Conflicts of Interest: All authors have completed the ICMJE uniform disclosure form (available at http://dx.doi. org/10.21037/tcr.2019.12.18). The authors have no conflicts of interest to declare.

Ethical Statement: The authors are accountable for all aspects of the work in ensuring that questions related to the accuracy or integrity of any part of the work are appropriately investigated and resolved. The study was conducted in accordance with the Declaration of Helsinki (as revised in 2013). This study was approved by the Second Hospital Review Board of Xi'an Jiaotong University (Xi'an, China). The approval number: 2019097. All cases were annotated with accessible clinical data to ensure the complete privacy and confidentiality of patient identity.

Open Access Statement: This is an Open Access article distributed in accordance with the Creative Commons Attribution-NonCommercial-NoDerivs 4.0 International License (CC BY-NC-ND 4.0), which permits the noncommercial replication and distribution of the article with the strict proviso that no changes or edits are made and the original work is properly cited (including links to both the formal publication through the relevant DOI and the license). See: https://creativecommons.org/licenses/by-nc-nd/4.0/.

\section{References}

1. Soldera SV, Leighl NB. Update on the Treatment of Metastatic Squamous Non-Small Cell Lung Cancer in New Era of Personalized Medicine. Front Oncol 2017;7:50.

2. Kamel HFM, Al-Amodi HSAB. Exploitation of Gene Expression and Cancer Biomarkers in Paving the Path to Era of Personalized Medicine. Genomics Proteomics Bioinformatics 2017;15:220-35.

3. Bais MV. Impact of Epigenetic Regulation on Head and Neck Squamous Cell Carcinoma. J Dent Res 2019;98:268-76.

4. Que SKT, Zwald FO, Schmults CD. Cutaneous squamous cell carcinoma: Incidence, risk factors, diagnosis, and staging. J Am Acad Dermatol 2018;78:237-47.

5. Chatterjee D, Bhagat R, Bansal V, et al. Parotid gland chondroma masquerading as pleomorphic adenoma in fine needle aspiration cytology: A diagnostic challenge. Diagn Cytopathol 2018;46:1060-3.

6. Montironi R, Cheng L, Lopez-Beltran A, et al. A low grade PIN-like neoplasm of the transition zone immunohistochemically negative for basal cell markers: a possible example of low grade adenocarcinoma with stratified epithelium. Pathology 2014;46:88-91.

7. Gandara DR, Hammerman PS, Sos ML, et al. Squamous cell lung cancer: from tumor genomics to cancer therapeutics. Clin Cancer Res 2015;21:2236-43.

8. McLean AEB, Barnes DJ, Troy LK. Diagnosing Lung Cancer: The Complexities of Obtaining a Tissue Diagnosis in the Era of Minimally Invasive and Personalised Medicine. J Clin Med 2018;7:163.

9. Rodriguez EF, Monaco SE, Dacic S. Cytologic subtyping of lung adenocarcinoma by using the proposed International Association for the Study of Lung Cancer/ American Thoracic Society/European Respiratory Society (IASLC/ATS/ERS) adenocarcinoma classification. Cancer Cytopathol 2013;121:629-37.

10. Takahashi Y, Eguchi T, Kameda K, et al. Histologic subtyping in pathologic stage I-IIA lung adenocarcinoma provides risk-based stratification for surveillance. Oncotarget 2018;9:35742-51.

11. Ujiie H, Kadota K, Chaft JE, et al. Solid Predominant Histologic Subtype in Resected Stage I Lung Adenocarcinoma Is an Independent Predictor of Early, Extrathoracic, Multisite Recurrence and of Poor Postrecurrence Survival. J Clin Oncol 2015;33:2877-84.

12. Biancosino C, Krüger M, Vollmer E, et al. Intraoperative fine needle aspirations-diagnosis and typing of lung cancer in small biopsies: challenges and limitations. Diagn Pathol 2016;11:59.

13. Mino-Kenudson M. Immunohistochemistry for predictive 
biomarkers in non-small cell lung cancer. Transl Lung Cancer Res 2017;6:570-87.

14. Birkman EM, Mansuri N, Kurki S, et al. Gastric cancer: immunohistochemical classification of molecular subtypes and their association with clinicopathological characteristics. Virchows Arch 2018;472:369-82.

15. Kadota K, Nitadori J, Rekhtman N, et al. Reevaluation and reclassification of resected lung carcinomas originally diagnosed as squamous cell carcinoma using immunohistochemical analysis. Am J Surg Pathol 2015;39:1170-80.

16. Cohen AS, Khalil FK, Welsh EA, et al. Cell-surface marker discovery for lung cancer. Oncotarget 2017;8:113373-402.

17. Kriegsmann K, Cremer M, Zgorzelski C, et al. Agreement of CK5/6, p40, and p63 immunoreactivity in non-small cell lung cancer. Pathology 2019;51:240-5.

18. Osmani L, Askin F, Gabrielson E, et al. Current WHO guidelines and the critical role of immunohistochemical markers in the subclassification of non-small cell lung carcinoma (NSCLC): Moving from targeted therapy to immunotherapy. Semin Cancer Biol 2018;52:103-9.

19. Pozzi C, Ferrari S, Luciani R, et al. Structural and Functional Characterization of the Human Thymidylate Synthase (hTS) Interface Variant R175C, New Perspectives for the Development of hTS Inhibitors. Molecules 2019;24:1362.

20. Niedzwiecki D, Hasson RM, Lenz HJ, et al. A Study of Thymidylate Synthase Expression as a Biomarker for Resectable Colon Cancer: Alliance (Cancer and Leukemia Group B) 9581 and 89803. Oncologist 2017;22:107-14.

21. Guo R, Tian Y, Jin X, et al. Thymidylate Synthase, a New Myoepithelial Biomarker for Breast Lesions. Int J Surg Pathol 2019;27:852-8.

22. Abdallah EA, Fanelli MF, Buim ME, et al. Thymidylate synthase expression in circulating tumor cells: a new tool to predict 5-fluorouracil resistance in metastatic colorectal cancer patients. Int J Cancer 2015;137:1397-405.

23. Intuyod K, Saavedra-García P, Zona S, et al. FOXM1

Cite this article as: Guo R, Tian Y, Zhang N, Huang H, Huang Y, Jin X, Huang X, Li Z, Yang J. Thymidylate synthase (TS) immunostaining in the diagnosis of the myoepithelial cells, basal cells, stratified epithelium cells, and associated tumors. Transl Cancer Res 2020;9(2):585-594. doi: 10.21037/ tcr.2019.12.18 modulates 5-fluorouracil sensitivity in cholangiocarcinoma through thymidylate synthase (TYMS): implications of FOXM1-TYMS axis uncoupling in 5-FU resistance. Cell Death Dis 2018;9:1185.

24. Lin CS, Liu TC, Lai JC, et al. Evaluating the Prognostic Value of ERCC1 and Thymidylate Synthase Expression and the Epidermal Growth Factor Receptor Mutation Status in Adenocarcinoma Non-Small-Cell Lung Cancer. Int J Med Sci 2017;14:1410-7.

25. Taddia L, D'Arca D, Ferrari S, et al. Inside the biochemical pathways of thymidylate synthase perturbed by anticancer drugs: Novel strategies to overcome cancer chemoresistance. Drug Resist Updat 2015;23:20-54.

26. Takeyama H, Wakasa $\mathrm{T}$, Inoue $\mathrm{K}$, et al. Thymidylate synthase expression in primary colorectal cancer as a predictive marker for the response to 5-fluorouracil- and oxaliplatin-based preoperative chemotherapy for liver metastases. Mol Clin Oncol 2018;9:3-10.

27. Burdelski C, Strauss C, Tsourlakis MC, et al. Overexpression of thymidylate synthase (TYMS) is associated with aggressive tumor features and early PSA recurrence in prostate cancer. Oncotarget 2015;6:8377-87.

28. Ferrari S, Severi L, Pozzi C, et al. Human Thymidylate Synthase Inhibitors Halting Ovarian Cancer Growth. Vitam Horm 2018;107:473-513.

29. Sulzyc-Bielicka V, Domagala P, Bielicki D, et al. Thymidylate synthase expression and p21(WAF1)/p53 phenotype of colon cancers identify patients who may benefit from 5-fluorouracil based therapy. Cell Oncol (Dordr) 2014;37:17-28.

30. Sulzyc-Bielicka V, Domagala P, Bielicki D, et al. E2F1/ TS Immunophenotype and Survival of Patients with Colorectal Cancer Treated with 5FU-Based Adjuvant Therapy. Pathol Oncol Res 2016;22:601-8.

31. Sasako $M$, Terashima $M$, Ichikawa $W$, et al. Impact of the expression of thymidylate synthase and dihydropyrimidine dehydrogenase genes on survival in stage II/III gastric cancer. Gastric Cancer 2015;18:538-48. 